\title{
Estado nutricional e hábitos alimentares de escolares de Campina Grande, Paraíba, Brasil
}

\author{
Nutritional status and eating habits of schoolchildren \\ in Campina Grande in the State of Paraíba, Brazil
}

Dixis Figueroa Pedraza ${ }^{1}$

Franciely Albuquerque Silva ${ }^{1}$

Nadinne Lívia Silva de Melo ${ }^{1}$

Erika Morganna Neves Araujo ${ }^{1}$

Carolina Pereira da Cunha Sousa ${ }^{1}$

${ }^{1}$ Programa de Pós-

Graduação em Saúde

Pública, Departamento de

Enfermagem, Universidade

Estadual da Paraíba. Av. das

Baraúnas 351, Bodocongó.

58109-753 Campina

Grande PB Brasil.

dixisfigueroa@gmail.com

\begin{abstract}
The nutritional status and its association with the eating habits of schoolchildren enrolled in public schools of Campina Grande in the State of Paraíba was evaluated. A cross-sectional study was conducted with 1081 children aged between 5 and10. Height/age (H/A) and body mass index/age (BMI/A) anthropometric indexes, as well as the eating habits of schoolchildren were analyzed. A prevalence of stunting, overweight and obesity of $2.4 \%, 12.3 \%$ and $9.2 \%$, respectively, was found. The H/A $z$-score revealed lower averages $(p=0.029)$ in children who reported almost never eating breakfast $(-0.130 \pm 1.053$ $z$-score) compared to children who eat this meal every day or almost every day $(0.183 \pm 0.912$ $z$-score); a similar association was observed when school meals were not eaten $(p=0.001)$. For the $B M I / A$, it was observed that children who have all meals had a lower average $z$-score $(p=0.034)$; similar results were observed in relation to the habit of eating breakfast, where a lower average value was found among children who reported this habit $(p=0.029)$. The nutritional status of schoolchildren is marked by high rates of overweight/obesity. This would suggest that losses in the nutritional status are related to failure to have breakfast and eat school meals.
\end{abstract}

Key words Nutritional status, Eating habits, Children
Resumo Avaliou-se o estado nutricional e sua associação com os hábitos alimentares de crianças escolares que frequentam a rede pública de ensino do município de Campina Grande, Paraíba. Estudo transversal, realizado com 1081 crianças de 5 a 10 anos. Analisaram-se os indices antropométricos estatura/idade (E/I) e Índice de Massa Corporal/idade (IMC/I), e os hábitos alimentares dos escolares. Verificaram-se prevalências de déficit de estatura, sobrepeso e obesidade de 2,4\%,12,3\% e $9,2 \%$, respectivamente. O E/I apresentou-se com média inferior $(p=0,029)$ nas crianças que indicaram quase nunca tomar café da manhã $(-0,130$ \pm 1,053 Escore-z) em comparação com as que faziam essa refeição todas ou na maioria das vezes (0,183 \pm 0,912 Escore-z), associação similar foi observada quando a merenda escolar não era consumida ( $p=0,001)$. Para o IMC/I, crianças com hábito de fazer todas as refeições apresentaram menor média ( $p=0,034)$; resultado similar foi observado em relação ao hábito de tomar café da manhã em que menor média foi constatada entre as crianças com esse costume $(p=0,020)$. Conclui-se que o estado nutricional dos escolares é marcado por altas taxas de sobrepeso/obesidade. Sugere-se que os prejuizos no estado nutricional estejam relacionados ao não consumo do café da manhã e da alimentação oferecida na escola.

Palavras-chave Estado nutricional, Hábitos alimentares, Criança 


\section{Introdução}

O crescimento físico é o parâmetro mais apropriado para avaliar as condições de saúde e o estado nutricional em crianças, por refletir a evolução da saúde e o desenvolvimento da população. Por este motivo, o desenvolvimento de pesquisas que objetivem o diagnóstico e o conhecimento dos problemas nutricionais, com vistas a melhorá-los para as próximas gerações, torna-se relevante ${ }^{1}$.

A desnutrição é responsável por acarretar importantes prejuízos na infância e representa a causa de mais de um terço das mortes infantis em todo o mundo ${ }^{2,3}$. Esse é um problema preocupante, uma vez que estima-se 178 milhões de crianças no mundo com baixa estatura, reflexo de alimentação insuficiente aliada à presença de doenças ${ }^{3}$. Além disso, a desnutrição infantil pode predispor a complicações na fase adulta ${ }^{2}$.

No Brasil persistem, ainda, formas severas de desnutrição, principalmente o comprometimento linear, o qual constitui importante problema de saúde pública. A situação é mais grave nas regiões Norte e Nordeste como resultado da desigualdade social e da distribuição de renda no país ${ }^{4,5}$. A maioria dos estudos sobre o tema tem sido realizada com menores de cinco anos, o que destaca a necessidade de pesquisas que envolvam crianças em idade escolar. Avaliar a desnutrição em escolares com enfoque direcionado ao déficit de estatura é importante por proporcionar uma visão ampliada em relação ao estado de saúde individual e coletivo ${ }^{6,7}$.

Observa-se, também, que entre as crianças de seis a dez anos de idade as prevalências de desnutrição infantil vêm diminuindo, enquanto que os casos de sobrepeso constituem condição nutricional em ascensão $0^{8,9}$. Essa condição é reflexo de mudanças nos hábitos alimentares dos indivíduos ${ }^{10,11}$, os quais são construídos durante a infância e se perpetuam por toda a vida ${ }^{12,13}$. Os hábitos inadequados associados ao estilo de vida não saudável, desde os primeiros anos de vida, intensificam os riscos de doenças crônicas na vida adulta ${ }^{14}$. Nesse sentido, a investigação precoce do consumo alimentar inadequado em crianças é necessária para que medidas de intervenção sejam realizadas o quanto antes ${ }^{15}$.

Os resultados da Pesquisa de Orçamentos Familiares (POF) ${ }^{16}$ apontaram taxa de obesidade de $18,3 \%$ entre os escolares brasileiros. Nesse mesmo estudo, o excesso de peso variou entre $25 \% \mathrm{e}$ $30 \%$, nas Regiões Norte e Nordeste, e entre 32\% e 40\%, nas Regiões Sul, Sudeste e Centro-Oeste. Além disso, a pesquisa apontou que a população brasileira vem sofrendo mudanças intensas no perfil de alimentação: diminuição no consumo de alimentos tradicionais e saudáveis, atrelada ao aumento da ingestão de alimentos industrializados.

A necessidade de maior quantidade de estudos sobre o sobrepeso/obesidade nas crianças e adolescentes brasileiros foi apontada como recomendação em trabalho recente de revisão sistemática sobre o tema ${ }^{17}$. Nessa revisão, 17 artigos foram elegíveis, indicando-se, ainda, maior carência nas Regiões Nordeste, Norte e Centro-Oeste, bem como maiores prevalências de sobrepeso no Sul e Nordeste. O trabalho em questão aborda, também, a relação estabelecida entre o sobrepeso e a obesidade com o desenvolvimento de hipertensão arterial sistêmica, dislipidemia e doenças cardio-metabólicas, dentre outras, o que reforça a importância de se direcionar esforços para melhorar o conhecimento das características epidemiológicas do sobrepeso/obesidade.

No presente estudo, objetivou-se avaliar o estado nutricional e sua associação com os hábitos alimentares de crianças escolares que frequentam a rede pública de ensino do município de Campina Grande, Paraíba.

\section{Metodologia}

O presente trabalho está vinculado ao projeto de pesquisa "Estado nutricional e Programa Nacional de Alimentação Escolar: perfil no contexto de escolas públicas municipais de Campina Grande-PB, para o desenvolvimento de um Programa de combate à fome e desnutrição". O projeto foi desenvolvido com o objetivo de avaliar o estado nutricional de escolares e o Programa de Alimentação Escolar na cidade de Campina Grande, Paraíba. A coleta de dados foi realizada em setembro de 2013.

O município de Campina Grande localiza-se na mesorregião do Agreste Paraibano e possui área total de $641 \mathrm{Km}^{2}$. Sua área urbana possui $98 \mathrm{Km}^{2}$ e a rural é de $420 \mathrm{Km}^{2}$. A população urbana de Campina Grande, segundo o Instituto Brasileiro de Geografia e Estatística, é de 337.484 habitantes e a rural de 17.847. Possui 51 bairros e cinco distritos, sendo três rurais (Galante, São José da Mata e Catolé de Boa Vista) e dois urbanos (Catolé de Zé Ferreira e Santa Terezinha).

Realizou-se estudo de delineamento transversal. No momento da coleta de dados, a Secretaria de Educação do município de Campina Grande, Paraíba, contava com 120 escolas de ensino funda- 
mental ( $1^{\circ}$ ciclo) que atendiam crianças de 5 a 10 anos de idade, distribuídas nas turmas de $1^{\circ}$ a $5^{\circ}$ ano. Localizavam-se 86 escolas na zona urbana ( 9 núcleos) e 34 na zona rural (4 núcleos), que atendiam, respectivamente, 21.696 e 2.801 escolares. A população elegível incluiu todas as crianças escolares, na faixa etária de 5 a 10 anos, de escolas urbanas da rede pública de ensino do município de Campina Grande e que frequentavam a escola no dia da coleta de dados, totalizando 1754 escolares.

Para garantir a representatividade das regiões e das diversidades administrativas do município, as escolas foram ordenadas segundo a distribuição por núcleos que adota o município. Em cada núcleo da zona urbana foram sorteadas duas escolas, totalizando 18. Em cada escola foi sorteada uma turma de cada ano, totalizando 90 turmas. Das crianças elegíveis, foram excluídos os escolares que não estavam na faixa etária (menores de cinco anos, do primeiro ano; e maiores de 10 anos, do quinto ano) e aqueles com problemas físicos que dificultassem a avaliação antropomé- trica. Além disso, houve perdas devido a escolares que não estavam presentes na escola ou não estavam acompanhados pelas mães no dia da coleta de dados e por dificuldades na avaliação antropométrica (criança muito inquieta ou chorando). Assim, do total de 1754 escolares, foram analisados 1093. Ainda, por incompletude das informações, 12 sujeitos foram declarados como perda, totalizando 1081 escolares de 5 a 10 anos de idade para o estudo. O fluxograma da seleção dos sujeitos da pesquisa é apresentado na Figura 1.

Os dados antropométricos foram coletados por uma equipe de alunos de graduação e pósgraduação da área de saúde da Universidade Estadual da Paraíba (UEPB). Os alunos foram treinados na UEPB para a padronização das medidas antropométricas e da coleta de dados por meio de questionário. Foi elaborado um manual de orientação para as medições da estatura e do peso seguindo as recomendações técnicas internacionais ${ }^{18}$. O treinamento foi ministrado pelo coordenador do projeto.

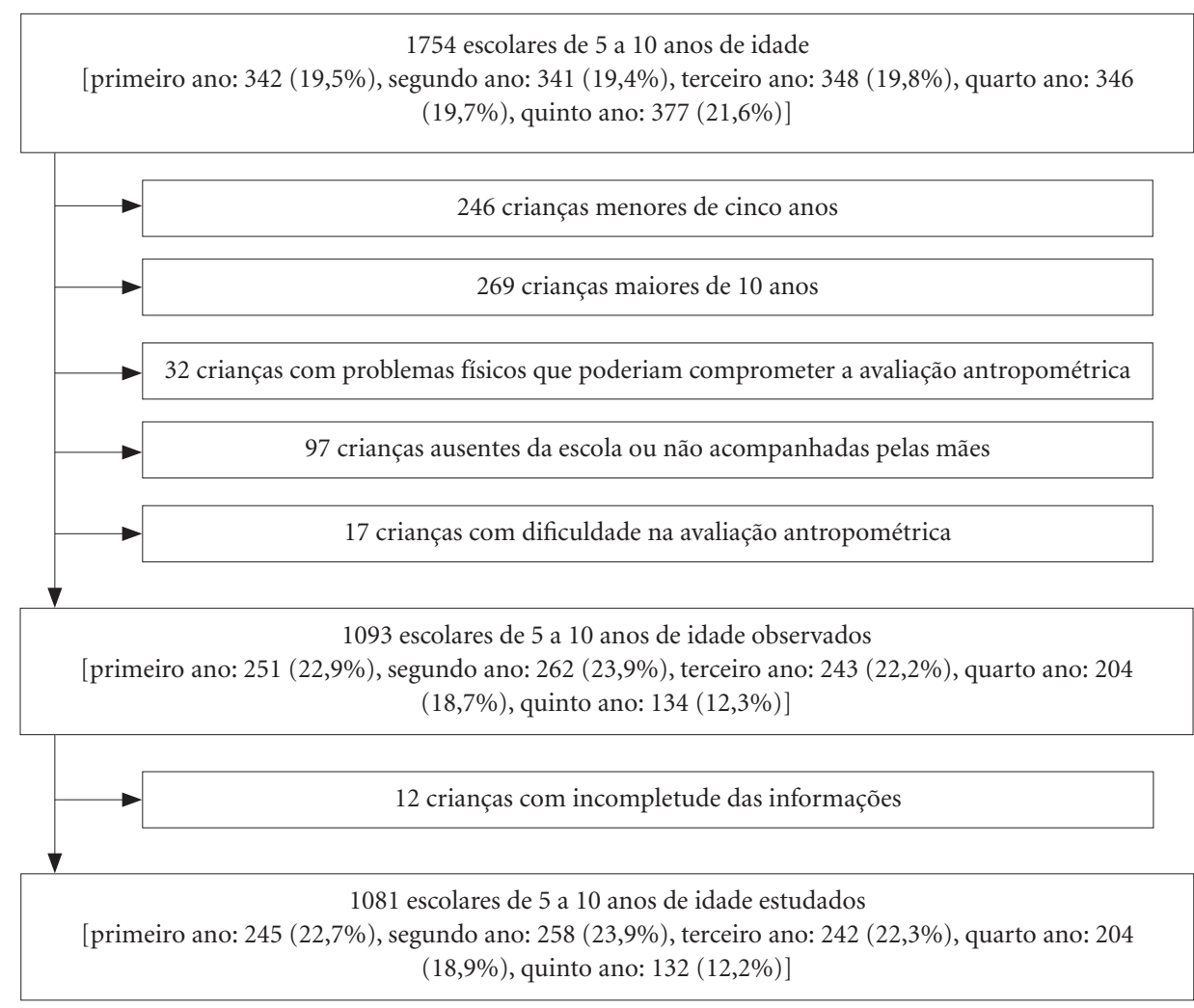

Figura 1. Fluxograma de seleção da população do estudo sobre o estado nutricional e os hábitos alimentares de escolares de 5 a 10 anos de idade. Campina Grande, Paraíba, Brasil, 2014. 
As informações obtidas, mediante entrevista realizada com as crianças, através do questionário previamente testado, incluíram as variáveis: sexo (feminino; masculino); idade em anos ( $<7 \mathrm{a}$; 7a-7a11m; 8a-8a11m; 9a-9a11m; 10a-10a11m); hábito de tomar café da manhã (quase nunca; às vezes; todas as manhãs ou a maioria delas); hábito de não realizar refeições (muitas vezes; poucas vezes; nunca); quantidade de frutas e verduras de ingestão diária (nenhuma; 1 a 3; 4 ou mais); alimentos de maior ingestão nos lanches (salgadinhos, batatas fritas e alimentos similares; sorvetes, doces, biscoitos doces/recheados, refrigerantes e alimentos similares; frutas, verduras e sucos de frutas); alimentação em casa (nunca tem fruta e verdura; às vezes tem fruta e verdura; sempre tem fruta e verdura); alimentação na merenda escolar (nunca tem fruta e verdura; às vezes tem fruta e verdura; sempre tem fruta e verdura); consumo diário da alimentação da merenda escolar (não; sim).

Os escolares tiveram a estatura medida por meio de estadiômetro (WCS ${ }^{\circledR}$ ) com amplitude de $200 \mathrm{~cm}$ e subdivisões de $0,1 \mathrm{~cm}$. As medições foram realizadas em duplicata, aceitando-se variação máxima de $3 \mathrm{~mm}$, e a medida final resultou da estimativa da média das duas medições. As crianças foram pesadas utilizando balança eletrônica do tipo plataforma com capacidade para $150 \mathrm{~kg}$ e graduação em $100 \mathrm{~g}$ (Tanita UM-080 ${ }^{\circledR}$ ).

Utilizaram-se os índices antropométricos Estatura/Idade (E/I) e Índice de Massa Corporal/ Idade (IMC/I). O estado nutricional das crianças foi expresso em escore-Z de acordo com o padrão de referência do Multicentre Growth Study (Who Reference 5-19 years $)^{19}$, atualmente recomendado pela Organização Mundial da Saúde. Os cálculos foram realizados com a utilização do programa WHO Anthro Plus 2009. Para a definição de baixa estatura foi adotado o ponto de corte E/I < Escore-z -2. Escolares com IMC/I $<$ Escore- $z-2$, $\geq$ Escore- $z-2$ e $<$ Escore- $z+1,>$ Escore- $z+1$ e $<$ Escore- $\mathrm{z}+2, \geq$ Escore- $\mathrm{z}+2$, foram classificados como de baixo IMC/I, eutrófico, sobrepeso, obeso, respectivamente ${ }^{20}$.

A digitação dos dados foi realizada imediatamente após a coleta da informação, em planilhas do programa Excel (Microsoft Inc., Estados Unidos) com opções de formatação e validação para a diminuição de erros. Amostra aleatória de 1/3 dos dados foi submetida à verificação da consistência considerando as informações contidas nos questionários. Discrepâncias, que foram corrigidas, encontraram-se em oito questionários.

A normalidade dos dados foi verificada por meio do teste de Kolmogorov-Smirnov. Para verificar diferenças nos Escore-z de E/I e IMC/I de acordo com o sexo, a idade e as variáveis relativas aos hábitos alimentares, realizaram-se análises com o uso do teste $t$-student, para variáveis de duas categorias, ou ANOVA, para variáveis de três ou mais categorias. Uma vez verificado os pressupostos da ANOVA, aplicou-se o teste F, com provas post-hoc de Bonferroni, para verificar diferenças entre as categorias. Todas as análises foram realizadas por meio do software estatístico $\mathrm{R}^{21}$, versão 3.1.2, adotando-se o nível de significância $<0,05$.

O projeto foi aprovado pelo Comitê de Ética em Pesquisa da Universidade Estadual da Paraíba. Foi solicitado consentimento escrito aos pais ou responsáveis pelas crianças e aos profissionais. Os resultados foram divulgados para as escolas e a Secretaria de Educação do município.

\section{Resultados}

O diagnóstico do estado nutricional dos 1081 escolares do estudo encontra-se representado na Gráfico 1. Verificou-se que 2,4\% (26) dos escolares estavam com déficit de estatura. $\mathrm{O}$ excesso de peso foi diagnosticado em 21,5\% (232) dos escolares, sendo $12,3 \%$ (133) de sobrepeso e 9,2\% (99) de obesidade.

A Tabela 1 apresenta a distribuição dos índices E/I e IMC/I de acordo com o sexo, a idade e os hábitos alimentares de escolares. Com relação aos hábitos alimentares, observou-se que $64,85 \%$ (701) dos escolares tomavam café da manhã todos os dias ou na maioria dos dias; 48,94\% (529) tinham o hábito de realizar todas as refeições; 12,21\% (132) não comiam frutas e/ou verduras; 46,99\% (508) lanchavam guloseimas, como sorvetes, doces, biscoitos doces/recheados e refrigerantes, e 22,20\% (240) lanchavam salgadinhos, batatas fritas ou alimentos similares. Quanto à alimentação de rotina em casa e na merenda escolar, as crianças indicaram a presença de frutas e verduras como a alternativa de maior frequência e o consumo diário da alimentação na escola por 63,74\% (689) delas.

Em relação aos índices antropométricos, média inferior $(p=0,029)$ para o $E / I$ encontrou-se nas crianças que quase nunca tomavam café da manhã $(-0,130 \pm 1,053)$ quando comparadas com aquelas que faziam essa refeição todas ou na maioria das vezes $(0,183 \pm 0,912)$. Para o IMC/I, destacou-se que crianças que indicaram o hábito de fazer todas as refeições apresentaram menor 
média do que as que responderam o contrário ( $\mathrm{p}$ $=0,034$ ), resultado similar em relação ao hábito de tomar café da manhã em que menor média foi constatada entre as crianças com esse costume $(\mathrm{p}=0,020)$. Além disso, crianças que não consumiam a merenda escolar apresentaram menor média de Escore-z de E/I do que crianças que responderam comer a merenda $(-0,168 \pm 1,028$ versus $0,078 \pm 1,047 ; \mathrm{p}=0,001)$.

\section{Discussão}

No presente estudo, as prevalências de déficit de estatura $(2,4 \%)$, sobrepeso $(12,3 \%)$ e obesidade $(9,15 \%)$ apresentaram-se inferiores às médias nacionais encontradas na POF $(6,8 \%, 33,5 \%$ e $14,3 \%$, respectivamente $)^{16}$. Apesar de este estudo apontar médias de sobrepeso e obesidade inferiores às encontradas nacionalmente, tais prevalências encontram-se elevadas e superiores quando comparadas ao déficit de estatura. Tais resultados caracterizam o processo de transição nutricional que, como consequência das melhores condições sociais, educativas e econômicas, vem acontecendo em várias partes do mundo, com destaque para as grandes cidades². No Brasil, esse padrão vem representado por prevalência de excesso de peso aproximadamente três vezes maior que a de

subnutrição $0^{22}$. Nos países da América-Latina, em geral, o processo de transição nutricional, caracteriza-se, ainda, pela coexistência do sobrepeso ou da obesidade com a subnutrição ${ }^{23}$.

O consumo de alimentos não saudáveis dos escolares de Campina Grande, inclusive dentro do ambiente escolar, evidenciada no presente estudo pelo consumo excessivo de alimentos ricos em açúcar como doces, guloseimas e refrigerantes, assemelha-se a resultados em outras localidades do país como em Belo Horizonte $(\mathrm{MG})^{12} \mathrm{e}$ em Chapada (RS) ${ }^{24}$, bem como em outros países como o Canadáa ${ }^{25} \mathrm{e}$ o México ${ }^{26}$. Analogamente, de acordo com a POF, os brasileiros estão cada vez mais substituindo o consumo de alimentos saudáveis, como cereais e hortaliças, por alimentos de alto teor energético, como refrigerantes e comidas prontas ${ }^{16}$. Esses dados remetem à importância da construção de hábitos alimentares saudáveis, por sua relevância tanto como fator de proteção em relação ao desenvolvimento de doenças, quanto de melhoria de condições de saúde na vida adulta quando adotadas as medidas apropriadas desde idades mais precoces ${ }^{13,27}$. Foi possível encontrar no presente estudo, ainda, frequências expressivas de crianças que omitiram o café da manhã e que deixaram de fazer refeições, assemelhando-se a constatações de estudos anteriores ${ }^{28-30}$.

O consumo diário da merenda escolar entre as crianças do presente estudo $(63,74 \%)$ foi inferior ao anteriormente observado com crianças de Piedade (SP) $(81,3 \%)^{14}$ e em Canoinhas (SC) $(95,7 \%)^{31}$. Argumenta-se que o consumo ou não da merenda escolar pode estar relacionada ao fato do estranhamento que pode causar, por serem os alimentos geralmente oferecidos no horário do almoço ou jantar, enquanto se associa a merenda a refeições leves e ao momento de brincadeiras ${ }^{32}$. Assim, há de reforçar a importância dos gestores e profissionais relacionados com a alimentação escolar de se preocuparem não apenas com a disponibilidade de alimentos para sua utilização na alimentação escolar, como também com mecanismos que viabilizem o consumo efetivo de tais alimentos para o qual a educação nutricional constitui uma ferramenta essencial ${ }^{33}$. Nesse sentido, o cardápio da merenda deve ser planejado de acordo com as influências culturais de cada região, no intuito de melhorar a sua aceitação, e a formação de hábitos alimentares saudáveis deve ser incentivada pelos profissionais envolvidos como as merendeiras e os nutricionistas ${ }^{33-35}$.

Pesquisadores em Itajaí (SC) e Indaial (SC) encontraram chance duas vezes maior de apre- 
Tabela 1. Distribuição dos índices estatura/idade (E/I) e índice de massa corporal/idade (IMC/I) de acordo com o sexo, a idade e os hábitos alimentares de escolares. Campina Grande, Paraíba, Brasil, 2014.

\begin{tabular}{|c|c|c|c|c|c|c|}
\hline \multirow{2}{*}{ Variáveis/Categorias } & \multirow[b]{2}{*}{$\mathbf{n}$} & \multirow[b]{2}{*}{$(\%)$} & \multicolumn{2}{|c|}{ E/I (Escore-z) } & \multicolumn{2}{|c|}{ ÍMC/I (Escore-z) } \\
\hline & & & $\begin{array}{c}\text { Média } \\
\text { (desvio-padrão) }\end{array}$ & $\mathbf{p}^{*}$ & $\begin{array}{c}\text { Média } \\
\text { (desvio-padrão) }\end{array}$ & $\mathbf{p}^{*}$ \\
\hline Sexo & & & & 0,555 & & 0,350 \\
\hline Feminino & 542 & 50,13 & $-0,057(1,049)$ & & $0,167(1,129)$ & \\
\hline Masculino & 539 & 49,87 & $-0,101(1,034)$ & & $0,226(1,279)$ & \\
\hline Idade & & & & 0,157 & & 0,240 \\
\hline$<7$ anos & 245 & 22,66 & $0,0008^{\mathrm{a}}(1,059)$ & & $0,271^{\mathrm{a}}(1,227)$ & \\
\hline $7-7$ anos e 11 meses & 258 & 23,87 & $-0,060^{\mathrm{a}}(1,058)$ & & $0,243^{\mathrm{a}}(1,221)$ & \\
\hline $8-8$ anos e 11 meses & 242 & 22,39 & $-0,149^{\mathrm{a}}(1,031)$ & & $0,092^{\mathrm{a}}(1,181)$ & \\
\hline $9-9$ anos e 11 meses & 204 & 18,87 & $-0,132^{\mathrm{a}}(0,995)$ & & $0,192^{\mathrm{a}}(1,261)$ & \\
\hline $10-10$ anos e 11 meses & 132 & 12,21 & $-0,053^{\mathrm{a}}(1,070)$ & & $0,161^{\mathrm{a}}(1,092)$ & \\
\hline Hábito de tomar café da manhã & & & & 0,029 & & 0,020 \\
\hline Quase nunca & 69 & 6,39 & $-0,130^{\mathrm{a}}(1,053)$ & & $0,530^{\mathrm{a}}(1,299)$ & \\
\hline Às vezes & 311 & 28,76 & $-0,021^{\mathrm{ab}}(1,035)$ & & $0,325^{\mathrm{ab}}(1,207)$ & \\
\hline Todas/maioria das manhãs & 701 & 64,85 & $0,183^{\mathrm{b}}(0,912)$ & & $0,106^{\mathrm{b}}(1,186)$ & \\
\hline Hábito de não realizar refeições & & & & 0,303 & & 0,034 \\
\hline Muitas vezes & 65 & 6,01 & $-0,085^{\mathrm{a}}(1,148)$ & & $0,256^{\mathrm{a}}(1,333)$ & \\
\hline Poucas vezes & 487 & 45,05 & $-0,016^{\mathrm{a}}(0,997)$ & & $0,320^{\mathrm{a}}(1,221)$ & \\
\hline Nunca & 529 & 48,94 & $-0,135^{\mathrm{a}}(1,067)$ & & $0,074^{\mathrm{b}}(1,165)$ & \\
\hline Quantidade de frutas e verduras por dia & & & & 0,804 & & 0,185 \\
\hline Nenhuma & 132 & 12,21 & $-0,069^{\mathrm{a}}(1,048)$ & & $0,119^{\mathrm{a}}(1,267)$ & \\
\hline 1 a 3 & 631 & 58,37 & $-0,087^{\mathrm{a}}(1,021)$ & & $0,244^{\mathrm{a}}(1,263)$ & \\
\hline 4 ou mais & 318 & 29,42 & $-0,065^{\mathrm{a}}(1,082)$ & & $0,131^{\mathrm{a}}(1,054)$ & \\
\hline Alimentos de maior ingestão nos lanches & & & & 0,054 & & 0,204 \\
\hline Salgadinhos, batatas fritas e similares & 240 & 22,20 & $-0,065^{\mathrm{ab}}(1,059)$ & & $0,229^{\mathrm{a}}(1,116)$ & \\
\hline $\begin{array}{l}\text { Sorvetes, doces, biscoitos doces/ } \\
\text { recheados, refrigerantes e similares }\end{array}$ & 508 & 46,99 & $-0,155^{\mathrm{b}}(1,034)$ & & $0,126^{\mathrm{a}}(1,172)$ & \\
\hline Frutas, verduras e suco de frutas & 333 & 30,81 & $0,027^{\mathrm{a}}(1,034)$ & & $0,278^{\mathrm{a}}(1,312)$ & \\
\hline Alimentação em casa & & & & 0,762 & & 0,449 \\
\hline Nunca tem fruta e verdura & 399 & 36,92 & $-0,144^{\mathrm{a}}(1,128)$ & & $0,127^{\mathrm{a}}(1,060)$ & \\
\hline Às vezes tem fruta e verdura & 594 & 54,94 & $-0,066^{\mathrm{a}}(1,048)$ & & $0,186^{\mathrm{a}}(1,234)$ & \\
\hline Sempre tem fruta e verdura & 88 & 8,14 & $-0,083^{a}(1,014)$ & & $0,226^{\mathrm{a}}(1,195)$ & \\
\hline Alimentação escolar & & & & 0,928 & & 0,527 \\
\hline Nunca tem fruta e verdura & 175 & 16,19 & $-0,166^{\mathrm{a}}(1,062)$ & & $0,107^{\mathrm{a}}(1,208)$ & \\
\hline Às vezes tem fruta e verdura & 665 & 61,52 & $-0,061^{\mathrm{a}}(1,042)$ & & $0,184^{\mathrm{a}}(0,184)$ & \\
\hline Sempre tem fruta e verdura & 241 & 22,29 & $-0,064^{\mathrm{a}}(1,027)$ & & $0,292^{\mathrm{a}}(1,359)$ & \\
\hline Consumo diário da alimentação escolar & & & & 0,001 & & 0,857 \\
\hline Não & 392 & 36,26 & $-0,168(1,028)$ & & $0,180(1,218)$ & \\
\hline Sim & 689 & 63,74 & $0,078(1,047)$ & & $0,224(1,186)$ & \\
\hline Total & 1081 & 100,0 & $-0,079(1,042)$ & & $0,196(1,206)$ & \\
\hline
\end{tabular}

* $\mathrm{p}$-valor do teste $t$-student para variáveis dicotômicas e ANOVA para variáveis não dicotômicas. ${ }^{\mathrm{a}, \mathrm{b}}$ Médias com diferentes letras em subscrito são significativamente diferentes, $\mathrm{p}<0,05$ (teste de Bonferroni).

sentar obesidade nos escolares que omitiam o café da manhã em relação àqueles que não tinham esse hábito ${ }^{13,30}$, convergindo com as médias superiores de IMC/I entre as crianças do presente estudo que não faziam a primeira refeição. De forma similar, tanto em Pelotas (RS), quanto nes- te trabalho, o hábito de não fazer refeições esteve associado ao desenvolvimento de obesidade ${ }^{36}$. Esses resultados foram sugeridos por meio de revisão sistemática que apontou o consumo do café da manhã e de maior número de refeições como fatores de proteção contra a obesidade en- 
tre crianças e adolescentes europeus ${ }^{37}$, bem como por outro estudo que sistematizou a proteção do consumo de cinco refeições ao dia em relação ao sobrepeso/obesidade entre crianças e adolescentes dos Estados Unidos, Alemanha e Portugal ${ }^{38}$. O café da manhã é uma refeição importante e não realizá-la pode prejudicar o desempenho escolar, a saciedade, o controle de peso e favorece o desejo de consumir lanches calóricos ${ }^{31,39}$. Por sua vez, o hábito de não fazer refeições deve ser extinto, pois o tempo prolongado entre as refeições favorece o desenvolvimento de gastrite e a ingestão maior de comida na próxima refeição para compensar o jejum ${ }^{40}$.

Em relação à $\mathrm{E} / \mathrm{I}$, os resultados deste estudo sugerem maior vulnerabilidade entre as crianças que não faziam a refeição do café da manhã e naquelas que não consumiam a merenda escolar. Esses resultados respaldam-se em princípios básicos do estado nutricional, ao se considerar o consumo alimentar insuficiente ou inadequado, juntamente com a presença de doenças, como fatores proximais do comprometimento do crescimento linear ${ }^{3,41}$. Crianças carentes têm, muitas vezes, na merenda escolar, a única refeição diária garantida, representando um atrativo e agindo como medida solucionadora para a necessidade básica de alimento ${ }^{31,42}$. Assim, a reduzida disponibilidade e/ou consumo de alimentos ao nível domiciliar (primeira refeição/café da manhã) ou escolar (merenda escolar), pode comprometer o aporte energético infantil e refletir no crescimento dessas crianças ${ }^{42,43}$. Além disso, os resultados sugerem-se relevantes, uma vez que, segundo revisão da literatura, nenhum estudo abordou, até o momento, a relação entre alimentação escolar e estado nutricional dos escolares ${ }^{33}$.

Outros fatores relacionados ao estado nutricional de escolares não foram considerados neste estudo. Nesse sentido, há que destacar, segundo os resultados da POF, o aumento da renda como determinante da diminuição do déficit de estatura e do aumento do sobrepeso e da obesidade nas crianças brasileiras de 5 a 9 anos de idade ${ }^{16}$. Em relação ao sobrepeso/obesidade, principal distúrbio nutricional deste estudo, crianças de cidade da região metropolitana de São Paulo apresentaram o desfecho associado à obesidade materna e a assistir televisão por quatro horas, além de ao consumo de alimentos não saudáveis ${ }^{44}$. Na conjuntura descrita, os resultados do presente estudo devem ser interpretados com cautela, pois as análises centraram sua atenção nos hábitos alimentares, questionando-se a possibilidade de confundimento por outras condições. Não obstante, cabe ressaltar a importância dos resultados apresentados considerando que as doenças não transmissíveis, associadas à obesidade e aos estilos de vida inadequados, constituem o lado mais negativo do panorama sanitário brasileiro atual ${ }^{45,46}$. Além disso, apesar de sugerir-se a idade escolar como a fase mais crítica da obesidade e reconhecer-se a importância da alimentação saudável na prevenção da doença ${ }^{44,46}$, as pesquisas não têm dado a devida atenção a essa relação no contexto escolar ${ }^{33}$.

Tem-se, ainda, como limitação do presente estudo seu delineamento transversal, impossibilitando o estabelecimento de relação causal por sua característica pontual. Vale ressaltar outra possível limitação relacionada à maturidade cognitiva dos escolares de fornecerem informações exatas e precisas. Assim, a interpretação dos resultados apresentados deve considerar que os mesmos podem estar influenciados por tais distinções.

\section{Conclusão}

O estado nutricional dos escolares apresentase com características da transição nutricional, marcada pelo aumento no indicador de sobrepeso/obesidade. Em face dos resultados, os hábitos alimentares dos escolares mostram-se associados ao estado nutricional, marcadamente, o não consumo do café da manhã como fator importante tanto no indicador de estatura/idade quanto no Índice de Massa Corporal/idade. Além disso, apresenta-se associação entre o consumo da alimentação escolar e o crescimento linear das crianças. Faz-se necessário, então, que o ambiente escolar funcione como veículo fornecedor de informações e atividades relacionadas a hábitos de alimentação saudável. A educação nutricional deve ser utilizada como estratégia para esses fins, mas também para promover maior aceitação da alimentação escolar. 


\section{Colaboradores}

D Figueroa Pedraza participou da elaboração do projeto, concepção do artigo, revisão bibliográfica, análise e interpretação dos dados, redação e aprovação do artigo. FA Silva participou da revisão bibliográfica, análise e interpretação dos dados, redação e aprovação do artigo. NLS Melo, EMN Araujo e CPC Sousa participaram da revisão bibliográfica, interpretação dos dados, redação e aprovação do artigo.

\section{Referências}

1. Borges GR, Mendes RCD, Moreira DOS. Estado nutricional de pré-escolares de escolas públicas e particulares de Dourados/MS. Interbio 2013; 7(2):67-78.

2. Vieira VL, Souza JMP, Cervato-Mancuso AM. Insegurança alimentar, vínculo mãe-filho e desnutrição infantil em área de alta vulnerabilidade social. Rev Bras Saúde Matern Infant 2010; 10(2):199-207.

3. Coutinho JG, Gentil PC, Toral N. A desnutrição e obesidade no Brasil: o enfrentamento com base na agenda única da nutrição. Cad Saude Publica 2008; 24(Supl. 2):332-340.

4. Eickhoff S, Nogueira LA. Acompanhamento de crianças desnutridas e fatores de risco: Uma parceria com a pastoral da criança. UNOPAR Cient Ciênc Biol Saude 2013; 15(1):55-58.

5. Oliveira FCC, Cotta RMM, Ribeiro AQ, Sant'Ana LFR, Priore SE, Franceschini SCC. Estado nutricional e fatores determinantes do déficit estatural em crianças cadastradas no Programa Bolsa Família. Epidemiol Serv Saúde 2011; 20(1):7-18.

6. Leal VS, Lira PIC, Menezes RCE, Oliveira JS, Sequeira LAS, Andrade SLLS, Batista Filho M. Fatores associados ao declínio do déficit estatural em crianças e adolescentes de Pernambuco. Rev Saude Publica 2012; 46(2):234241.

7. Guedes DP, Mendes RR. Crescimento físico e estado nutricional de escolares do Vale do Jequitinhonha, Minas Gerais, Brasil. Rev Bras Cineantropom Desempenho Hum 2012; 14(4):363-376.

8. Rodrigues PA, Marques MH, Chaves MGAM, Souza CF, Carvalho MF. Prevalência e fatores associados a sobrepeso e obesidade em escolares da rede pública. Cien Saude Colet 2011; 16(Supl. 1):1581-1588.

9. Reis CEG, Vasconcelos IAL, Oliveira OMV. Panorama do estado antropométrico dos escolares brasileiros. Rev Paul Pediatr 2011; 29(1):108-116.

10. Felisbino-Mendes MS, Campos MD, Lana FCF. Avaliação do estado nutricional de crianças menores de 10 anos no município de Ferros, Minas Gerais. Rev Esc Enferm USP 2010; 44(2):257-265.

11. Ferreira HS, Luciano SCM. Prevalência de extremos antropométricos em crianças do estado de Alagoas. Rev Saude Publica 2010; 44(2):377-380.

12. Carvalho AP, Oliveira VB, Santos LC. Hábitos alimentares e práticas de educação nutricional: atenção a crianças de uma escola municipal de Belo Horizonte, Minas Gerais. Pediatria (São Paulo) 2010; 32(1):20-27.

13. Bertin RL, Malkowski J, Zutter LCI, Ulbrich AZ. Estado nutricional, hábitos alimentares e conhecimento de nutrição em escolares. Rev Paul Pediatr 2010; 28(3):303-308.

14. Pegolo GE, Silva MV. Consumo de energia e nutrientes e a adesão ao Programa Nacional de Alimentação Escolar (PNAE) por escolares de um município paulista. Segur Alim Nutr 2010; 17(2):50-62.

15. Hinnig PF, Bergamaschi DP. Itens alimentares no consumo alimentar de crianças de 7 a 10 anos. Rev Bras Epidemiol 2012; 15(2):324-334.

16. Instituto Brasileiro de Geografia e Estatística (IBGE) Pesquisa de Orçamentos Familiares 2008-2009: Antropometria e estado nutricional de crianças, adolescentes e adultos no Brasil. Rio de Janeiro: IBGE; 2010. 
17. Niehues JR, Gonzales AI, Lemos RR, Bezerra PP, Haas P. Prevalence of Overweight and Obesity in Children and Adolescents from the Age Range of 2 to 19 Years Old in Brazil. Int J Pediatr 2014; 2014:583207.

18. World Health Organization (WHO). Physical status: the use and interpretation of anthropometry: report of an Expert Committee. Technical Report Series. Geneva: WHO; 1995

19. Onis M, Onyango AW, Borghi E, Siyam A, Nishida C, Siekmanna J. Development of a WHO growth reference for school-aged children and adolescents. Bull World Health Org 2007; 85(9):660-667.

20. Brasil. Ministério da Saúde (MS). Orientações para a coleta e análise de dados antropométricos em serviços de saúde: Norma Técnica do Sistema de Vigilância Alimentar e Nutricional - SISVAN. Brasília: MS; 2011 (Série G. Estatística e Informação em Saúde).

21. R Development Core Team. R: A Language and Environment for Statistical Computing [computer program]. Version 3.1.2. Vienna: R Foundation for Statistical Computing; 2011.

22. Conde WL, Monteiro CA. Nutrition transition and double burden of undernutrition and excess of weight in Brazil. Am J Clin Nutr 2014; 100(Supl. 6):1617-1622.

23. Rivera JA, Pedraza LS, Martorell R, Gil A. Introduction to the double burden of undernutrition and excess weight in Latin America. Am J Clin Nutr 2014; 100(Supl. 6):1613-1616.

24. Polla SF, Scherer F. Perfil alimentar e nutricional de escolares da rede municipal de ensino de um município do interior do Rio Grande do Sul. Cad Saúde Colet 2011; 19(1):111-116.

25. Mâsse LC, Niet-Fitzgerald JE, Watts AW, Naylor PJ, Saewyc EM. Associations between the school food environment, student consumption and body mass index of Canadian adolescents. Int J Behav Nutr Phys Act 2014; 11(29):1-9.

26. Moraes SA, Beltrán RJ, Mondini L, Freitas ICM. Prevalência de sobrepeso e obesidade e fatores associados em escolares de área urbana de Chilpancingo, Guerrero, México, 2004. Cad Saude Publica 2006; 22(6):12891301.

27. Conceição SIO, Santos CJN, Silva AAM, Silva JS, Oliveira TC. Consumo alimentar de escolares das redes pública e privada de ensino em São Luís, Maranhão. Rev Nutr 2010; 23(6):993-1004.

28. Shafiee G, Kelishadi R, Qorbani M, Motlagh ME, Taheri M, Ardalan G, Taslimi M, Poursafa P, Heshmat R, Larijani B. Association of breakfast intake with cardiometabolic risk factors. J Pediatr (Rio J) 2013; 89(6):575-582.

29. Couto SF, Madruga SW, Neutzling MB, Silva MC. Frequência de adesão aos "10 Passos para uma Alimentação Saudável” em escolares adolescentes. Cien Saude Colet 2014; 19(5):1589-1599.

30. Hofelmann DA, Momm N. Café da manhã: omissão e fatores associados em escolares de Itajaí, Santa Catarina, Brasil. Nutrire 2014; 39(1):40-55.

31. Mota CH, Mastroeni SSBS, Mastroeni MF. Consumo da refeição escolar na rede pública municipal de ensino. $R$ Bras Est Pedag 2013; 94(236):168-194.
32. Freitas MCS, Minayo MCS, Ramos LB, Fontes GV, Santos LA, Souza EC, Santos AC, Mota SE, Paiva JB, Bernardelli TM, Demétrio F, Menezes I. Escola: lugar de estudar e de comer. Cien Saude Colet 2013; 18(4):979985.

33. Oliveira MC, Vassimon HS. Programa Nacional de Alimentação Escolar e sua aceitação pelos alunos: uma revisão sistemática. Investigação 2012; 12(1):4-10.

34. Chaves LG, Santana TCM, Gabriel CG, Vasconcelos FAG. Reflexões sobre a atuação do nutricionista no Programa Nacional de Alimentação Escolar no Brasil. Cien Saude Colet 2013; 18(4):917-926.

35. Fernandes AGS, Fonseca ABC, Silva AA. Alimentação escolar como espaço para educação em saúde: percepção das merendeiras do município do Rio de Janeiro, Brasil. Cien Saude Colet 2014; 19(1):39-48.

36. Terres NG, Pinheiro RT, Horta BL, Pinheiro KAT, Horta LL. Prevalência e fatores associados ao sobrepeso e à obesidade em adolescentes. Rev Saude Publica 2006; 40(4):627-633.

37. Szajewskaa H, Ruszczyńskia M. Systematic Review Demonstrating that Breakfast Consumption Influences Body Weight Outcomes in Children and Adolescents in Europe. Crit Rev Food Sci Nutr 2010; 50(2):113-119.

38. Koletzko B, Toschke AM. Meal patterns and frequencies: do they affect body weight in children and adolescents? Crit Rev Food Sci Nutr 2010; 50(2):100-105.

39. Trancoso SC, Cavalli SB, Proença RPC. Café da manhã: caracterização, consumo e importância para a saúde. Rev Nutr 2010; 23(5):859-869.

40. Silva JG, Teixeira MLO, Ferreira MA. Alimentação e saúde: sentidos atribuídos por adolescentes. Esc Anna Nery 2012; 16(1):88-95.

41. Figueroa Predraza D, Rocha ACD, Sousa CPC. Crescimento e deficiências de micronutrientes: perfil das crianças assistidas no núcleo de creches do governo da Paraíba, Brasil. Cien Saude Colet 2013; 18(11):33793390.

42. Souza ALC, Mamede MEO. Estudo sensorial e nutricional da merenda escolar de uma escola da cidade de Lauro de Freitas - BA. Rev Inst Adolfo Lutz 2010; 69(2):255-260

43. Antunes MML, Schieri R, Salles-Costa R. Consumo alimentar de crianças menores de três anos residentes em área de alta prevalência de insegurança alimentar domiciliar. Cad Saude Publica 2010; 26(8):1642-1650.

44. Mondini L, Levy RB, Saldiva SRDM, Venâncio SI, Aguiar JA, Stefanini MLR. Overweight, obesity and associated factors in first grade schoolchildren in a city of the metropolitan region of São Paulo, Brazil. Cad Saude Publica 2007; 23(8):1825-1834.

45. Minayo MCS. Hipertensão, diabetes, obesidade e outros males do Brasil contemporâneo. Cien Saude Colet 2014; 19(6):1640-1641.

46. Ferrari HG. Panorama da obesidade em crianças e adolescentes brasileiros: revisão dos últimos 10 anos. Pediatria (São Paulo) 2009; 31(1):58-70.

Artigo apresentado em 19/09/2015

Aprovado em 26/04/2016

Versão final apresentada em 28/04/2016 
